\title{
Skin infection with Saccharomyces cerevisiae in an immunocompetent patient: An exceptional infection
}

\section{Siham Belmourida, Hind Palamino, Mariame Meziane, Nadia Ismaili, Laila Benzekri, Badredine Hassam, Karima Senouci}

Department of Dermatology-Venerology, Mohammed V University, Ibn Sina Hospital, Rabat, Morocco.

Corresponding author: Dr. Siham Belmourida, E-mail: belmouridasiham2017@gmail.com

Sir,

Saccharomyces cerevisiae is a ubiquitous yeast used as a probiotic for antibiotic-associated diarrhea prophylaxis. It is part of the normal flora of the oral, gastrointestinal, and respiratory tracts and the vaginal lining. Severe opportunistic infections due to $S$. cerevisiae have been reported in patients with immunosuppression with fungemia, endocarditis, pneumonia, and urinary tract infection [1-3].

Herein, we describe the first case in the literature of a skin infection with S. cerevisiae in an immunocompetent patient.

A 72-year-old patient with no particular history had had pruriginous skin lesions for five years in the context of general state preservation and apyrexia.

A skin examination revealed erythematous and maculopapular lesions diffused throughout the body (Figs. la-ld). The phaners and mucous membranes were without abnormality, and there was no adenopathy. The rest of the examination was unremarkable. The patient had been treated several times with topical and oral antifungals and dermocorticoids but without any improvement.

Biological examination revealed no immunodepression. Two mycological examinations of the skin lesions revealed the presence of $S$. cerevisiae (Figs. 2 and 3). Unfortunately, the patient proceeded against medical advice and discontinued attendance without treatment.
The genus Saccharomyces comprises several species, with the most well-known being $S$. cerevisiae.

Saccharomyces cerevisiae is a common colonizer of mucous surfaces and part of the normal flora of the gastrointestinal tract, respiratory tract, and vagina [1]. Invasive $S$. cerevisiae infections have been identified for the past three decades, with the microorganisms recovered from the blood, lungs, esophagi, peritoneal cavities, urinary tracts, and vaginas. Clinical and commercial strains are being identified with genotyping studies, and the contribution of commercial strains to the colonization and infection has been documented [1]. The predisposing risk factors are similar to those associated with invasive candidiasis. The treatment of choice has not been established: $S$. cerevisiae is persistently sensitive to amphotericin $\mathrm{B}$ and fluorocytosine while resistant to fluconazole and itraconazole. A literature review by Munoz et al. reported a favorable response in $60 \%$ of 19 patients with invasive $S$. cerevisiae who received fluconazole alone, in contrast to a favorable response in $77.7 \%$ of 31 patients receiving amphotericin B [2]. The role of echinocandins has not yet been established and was not reported in the study $[1,2]$.

Ours is the first case of $S$. cerevisiae discovered in an immunocompetent patient, whose immunodepression might be explained by advanced age; however, it remains uncertain whether this is necessary for a skin infection with $S$. cerevisiae.

Reports of Saccharomyces cerevisiae infections in dermatological publications are nonexistent, and, to

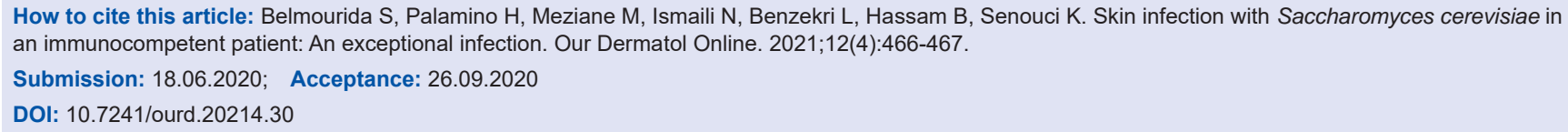



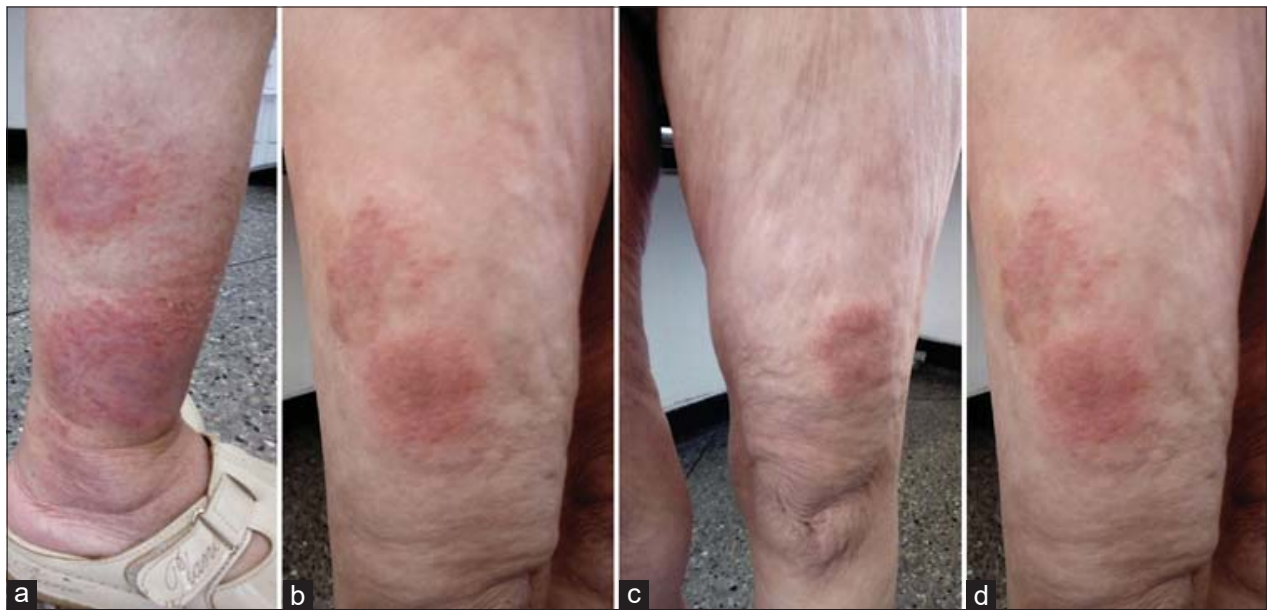

Figure 1: (a-d) Erythematous maculopapular lesions of the lower members.

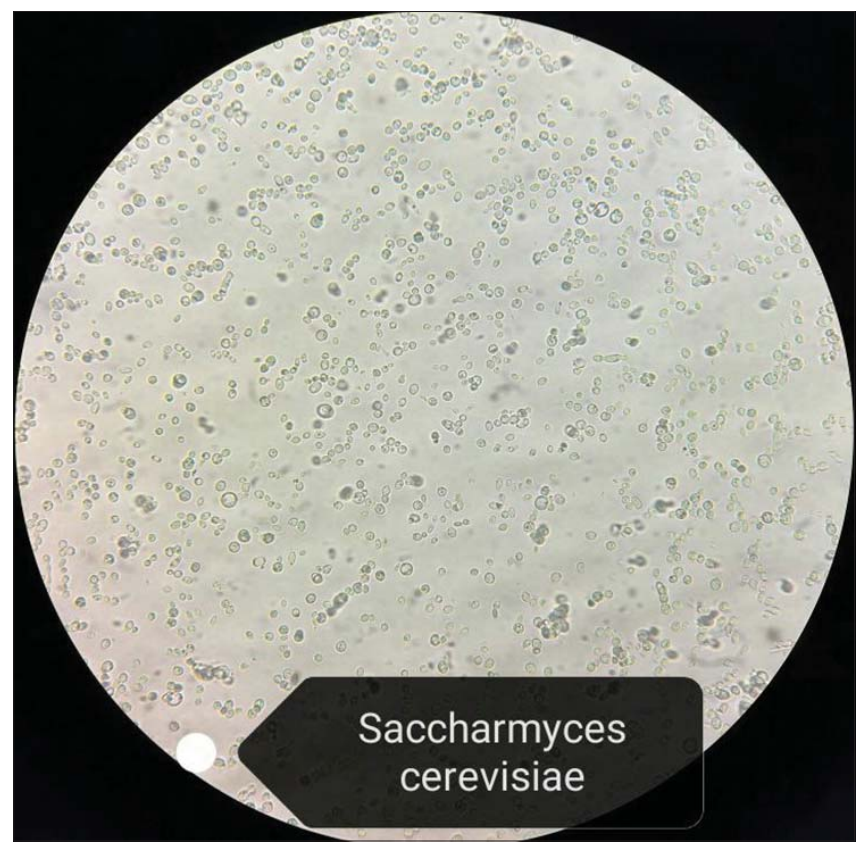

Figure 2: A microscopic image highlighting the arrangement of Saccharomyces cerevisiae yeast.

date, they remain exceptional. Studies are needed to elucidate their pathophysiological mechanism as it still remains poorly understood.

Although skin infections with $S$. cerevisiae remain exceptional to this day, it is preferable to perform a cultured mycological examination in front of ring lesions resistant to the usual treatments, especially in elderly persons.

\section{Consent}

The examination of the patient was conducted according to the principles of the Declaration of Helsinki.

The authors certify that they have obtained all appropriate patient consent forms, in which the patients have given consent for images

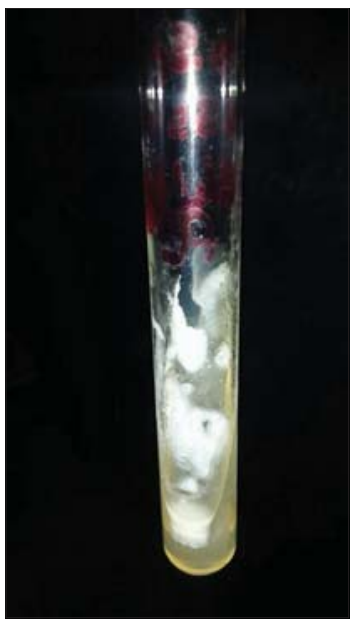

Figure 3: Saccharomyces cerevisiae.

and other clinical information to be included in the journal. The patients understand that their names and initials will not be published and due effort will be made to conceal their identity, but that anonymity cannot be guaranteed.

\section{REFERENCES}

1. Fadhel M, Patel S, Liu E, Levitt M, Asif A. Saccharomyces cerevisiae fungemia in a critically ill patient with acute cholangitis and long term probiotic use. Med Mycol Case Rep. 2019;23:23-5.

2. Muñoz P, Bouza E, Cuenca-Estrella M, Eiros JM, Pérez MJ, Sánchez-Somolinos M, et al. Saccharomyces cerevisiae fungemia: An emerging infectious disease. Clin Infect Dis. 2005;40:1625-34.

3. Atıcı S, Soysal A, Karadeniz Cerit K, Yılmaz Ş, Aksu B, Kıyan G, et al. Catheter-related Saccharomyces cerevisiae fungemia following Saccharomyces boulardii probiotic treatment: in a child in intensive care unit and review of the literature. Med Mycol Case Rep. 2017;15:33-5.

Copyright by Siham Belmourida, et al. This is an open-access article distributed under the terms of the Creative Commons Attribution License, which permits unrestricted use, distribution, and reproduction in any medium, provided the original author and source are credited.

Source of Support: Nil, Conflict of Interest: None declared. 